\title{
1 Reduced Molecular Flavins as Single-Electron Reductants after 2 Photo-Excitation
}

3 Richard Foja, Alexandra Walter, Christian Jandl, Erling Thyrhaug, Jürgen Hauer, and Golo Storch*

4 Department of Chemistry, Technical University of Munich, Lichtenbergstr. 4, 85747 Garching, Germany

5 E-mail: golo.storch@tum.de

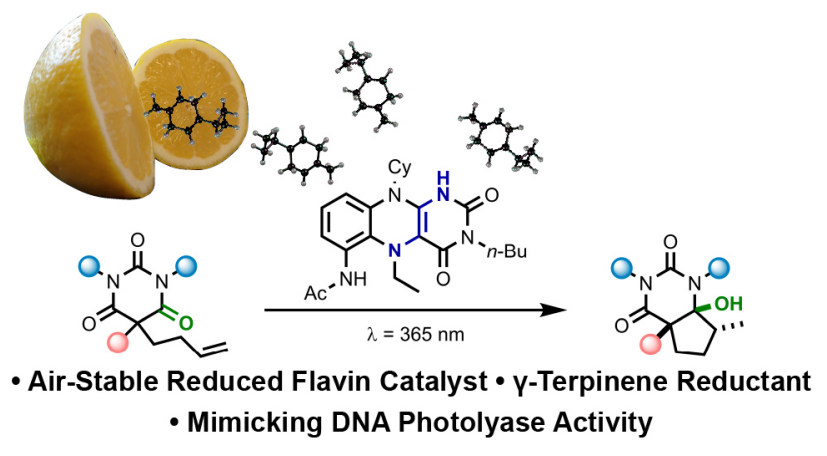

9 Abstract

10 Flavoenzymes mediate a multitude of chemical reactions and are catalytically active both in different oxidation 11 states and in covalent adducts with reagents. The transfer of such reactivity to the organic laboratory using simpli12 fied molecular flavins is highly desirable and such applications in (photo-)oxidation reactions are already estab13 lished. However, molecular flavins have not been used for the reduction of organic substrates yet, although this 14 activity is known and well-studied for DNA photolyase enzymes. We report a catalytic method using reduced, 15 molecular flavins as photo-reductants and $\gamma$-terpinene as sacrificial reductant. Additionally, we present our design 16 for air-stable, reduced flavin catalysts, which is based on a conformational bias strategy and circumvents the oth17 erwise rapid reduction of $\mathrm{O}_{2}$ from air. Using our catalytic strategy, we were able to replace super-stoichiometric 18 amounts of the rare-earth reductant $\mathrm{SmI}_{2}$ in a 5-exo-trig cyclization of substituted barbituric acid derivatives. Such 19 flavin-catalyzed reductions are anticipated to be of broad applicability and their straightforward synthesis indicates future use in stereo- as well as site-selective transformations.

Main Text

Flavins are versatile cofactors in enzymes and are involved in a variety of chemical transformations either as flavin adenine dinucleotide (FAD) or flavin mononucleotide (FMN). ${ }^{1}$ This diversity stems from the occurrence of different catalytically active cofactor states and depends on oxidation or reduction (FAD vs. $\mathrm{FADH}_{2}$ ) as well as photochemical excitation. Among the known reactions of flavoenzymes, the cleavage of thymine dimers 1 by DNA photolyase 
is a particularly interesting and relevant reaction, which is mediated by excited, hydroquinoid $\mathrm{FADH}^{\mathbf{-}^{*}}\left(\mathbf{2}^{-*}\right)$. Thymine dimers $(\mathrm{T}<>\mathrm{T}) \mathbf{1}$ are the result of UV-light mediated [2+2]-cycloaddition reaction between adjacent thymines in DNA and their formation hampers correct transcription or replication. The enzymatic strategy to revert such DNA damage relies on the excitation of the reduced cofactor $2^{-}$and subsequent one-electron reduction of $(\mathrm{T}<>\mathrm{T})$ 1 (Figure 1). ${ }^{2}$ The ketyl radical intermediate $\mathbf{1}^{--}$undergoes rapid cyclobutane ring-opening and back electron transfer to the semiquinone $\mathbf{2}^{\circ}$, which leads to the release of both thymines and closes the catalytic cycle. The very negative redox potential $E\left(\mathbf{1}^{-\mathbf{1}^{-}}\right)=-2.2 \mathrm{~V} v \mathrm{~s}$. SCE (in $\left.\mathrm{CH}_{3} \mathrm{CN}\right)$ of thymine dimer 1 highlights the strong reducing capacity of excited cofactor $2^{-*}{ }^{3}$ Despite the great potential for synthetic transformations, such one-electron reductions have not yet been applied using molecular flavin catalysts in the organic laboratory, although the photosensitized $^{4}$ and oxidative ${ }^{5}$ cleavage of thymine dimers with molecular flavins has been studied. The general usefulness of reduced flavin catalysts is highlighted by recent reductive catalytic reactions with flavoenzymes ${ }^{6}$ and a deazaflavin. ${ }^{7}$ We found it remarkable, that in organic synthesis molecular flavins are applied for oxidations extensively, ${ }^{8}$ for example using commercially available (-)-riboflavin, ${ }^{9}$ but not for reductions. Therefore, we aimed for finding a strategy to use molecular flavins in the reductive 5-exo-trig cyclization of barbituric acid derivatives, which had previously only been reported with super-stoichiometric metal reductants (Figure 1B). ${ }^{10,11}$
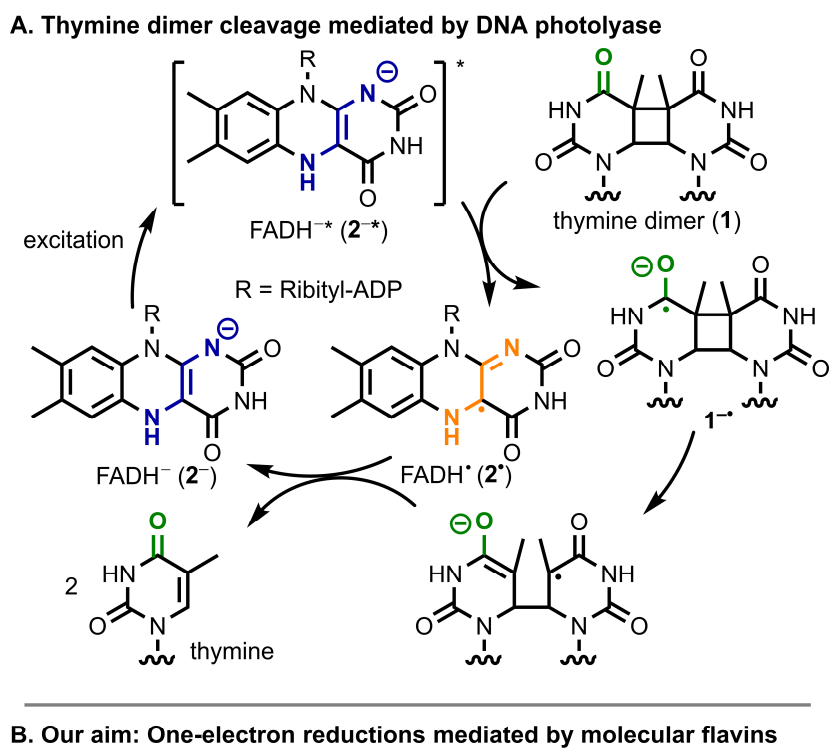

Figure 1. Mechanism of enzymatic thymine dimer 1 cleavage mediated by DNA photolyase (A). In the key step, an electron is transferred from the excited cofactor $\mathbf{2}^{-*}$ to the dimerized thymine $\mathbf{1}$. Our envisioned transformation using molecular, reduced flavin catalysts relies on similar one-electron transfer (B). Carbonyl groups involved in reduction are highlighted in green.

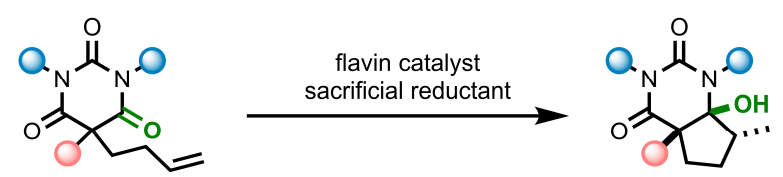


We hypothesized that the instability of reduced flavins such as $\mathbf{2}$ towards oxygen from air is one reason for the above-described discrepancy since it renders studies of the reduced cofactor very impractical. The instability itself is the result of a rapid reduction of $\mathrm{O}_{2}$ to $\mathrm{O}_{2}{ }^{-}$and subsequent formation of covalently bound flavin hydroperoxides. ${ }^{12}$ The initial reduction of molecular oxygen $\left[E\left(\mathrm{O}_{2} / \mathrm{O}_{2}^{-\cdot}\right)=-0.55 \mathrm{~V} v\right.$ s. SCE $]$ by a reduced flavin $\left[E\left(\mathrm{Fl}^{\cdot+} / \mathrm{Fl}\right)=-0.05\right.$ $\mathrm{V} v s . \mathrm{SCE}$ ] (both values in aqueous buffer at $\mathrm{pH}=4.6$ ) is disfavored, but the net reaction becomes exergonic upon formation of the thermodynamically favored oxidized flavin and hydrogen peroxide. ${ }^{13}$ We decided to base our strategy for air-stable, reduced flavin catalysts on a conformational bias for the reduced form, ${ }^{14}$ which is typically bent along the N5-N10 axis with a ring puckering angle of $27.3^{\circ} .{ }^{15}$ Both oxidized, as well as semiquinone states, are (almost) planar. ${ }^{16}$ The conformational bias was achieved by double substitution of the N5- and C6-positions via reductive alkylation of flavin $\mathbf{3}$ and reduced catalyst $\mathbf{4}$ was obtained as an air-stable solid (Figure 2A). However, unsubstituted analog $\mathbf{5}$ did not yield the reduced flavin and was instead oxidized by air. Both oxidized flavins have similar redox properties: $E_{1 / 2}=-0.78 \mathrm{~V} v s$. SCE (3) and $E_{1 / 2}=-0.85 \mathrm{~V} v s$. SCE (5). The bent structure of flavin 4 (Figure 2B) was further characterized by single-crystal diffraction and a ring puckering angle of $32.1^{\circ}$ was found along the N5-N10 axis (Figure $2 \mathrm{C}$ ). Cyclic voltammetry confirmed that oxidation $\left[E_{1 / 2}=+0.46 \mathrm{~V} v s\right.$. SCE (4) in $\mathrm{CH}_{3} \mathrm{CN}$ ] of reduced flavin 4 is at least partially reversible (Figure $2 \mathrm{C}$ ). When the measurement was continued to more positive potentials, irreversible processes were detected which is in line with our strategy of destabilizing the planar oxidized states.



B

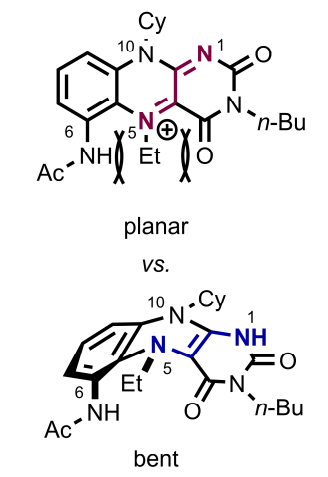

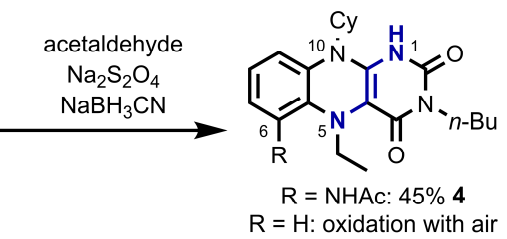

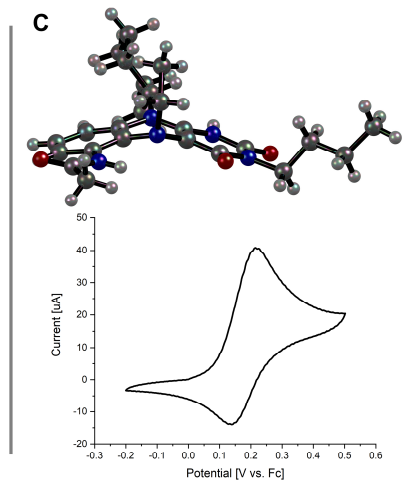

Figure 2. Synthesis and characterization of an air-stable reduced flavin. Conformational bias is realized by reductive alkylation (A) which results in steric destabilization of the planar states (B). The bent structure of flavin 4 is visible in the $\mathrm{X}$-ray structure $(\mathrm{C})$ and cyclic voltammetry in $\mathrm{CH}_{3} \mathrm{CN}$ shows partially reversible electron transfer.

We then turned our attention to the photophysical properties of air-stable flavin $\mathbf{4}$ and its oxidized counterpart 3 (Figure 3). The first major difference is the blue-shift of the absorption maximum, which is at $\lambda_{\mathrm{A}, \max }=324 \mathrm{~nm}$ for the reduced catalyst. The same trend is observed for the emission with fluorescence occurring at $\lambda_{\mathrm{E}, \max }=567 \mathrm{~nm}$. 
In analogy to the natural cofactor $\left(\mathrm{FADH}_{2} v s . \mathrm{FADH}^{-}\right)$, the emission of 4 is shifted to $\lambda_{\mathrm{E}, \max }=533 \mathrm{~nm}$ upon addition of triethylamine base. ${ }^{2 c}$ We observed two long lifetime components of flavin 4 in the excited state (see SI): $\tau=$ $1.1 \mathrm{~ns}(78 \%)$ and $\tau=11.7 \mathrm{~ns}(22 \%)$.
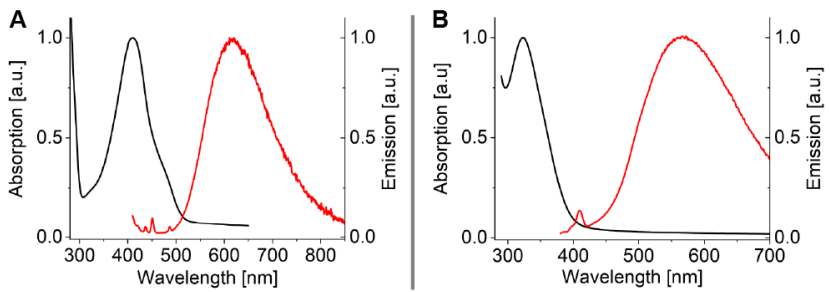

Figure 3. Normalized absorption (in black) and emission (in red) spectra of flavin 3 (A) and reduced flavin 4 (B) 81 in $\mathrm{CH}_{3} \mathrm{CN}$ solution. Quinoid flavin 3: $\lambda_{\mathrm{A}, \max }=411 \mathrm{~nm} ; \lambda_{\mathrm{E}, \max }=620 \mathrm{~nm}$. Hydroquinoid flavin 4: $\lambda_{\mathrm{A}, \max }=324 \mathrm{~nm}$; $\lambda_{\mathrm{E}, \max }=567 \mathrm{~nm}$.

83

84 With this information in hand, we started our catalysis studies and chose barbituric acid derivative 6 as a promising substrate for one-electron reduction due to its structural and electronic similarity to thymine dimer $\mathbf{1}$. The 5-exotrig cyclization of substrate 6 had previously been reported using six equivalents of rare-earth reductant $\mathrm{SmI}_{2},{ }^{10}$ thus making this reaction an ideal target for a mild catalytic protocol. Optimization of the catalytic conditions (see SI for details) quickly revealed that $\mathbf{4}$ is indeed a suitable electron donor in the excited state. Several sacrificial reductants turned out to be applicable, but we decided to move forward with $\gamma$-terpinene which is an inexpensive and commercially available essential oil. ${ }^{17}$ With a catalytic amount of triethylamine and a catalytic amount of cysteine as a hydrogen atom donor, ${ }^{18}$ we obtained bicycle 7 in 90\% yield (Table 1, Entry 1). 

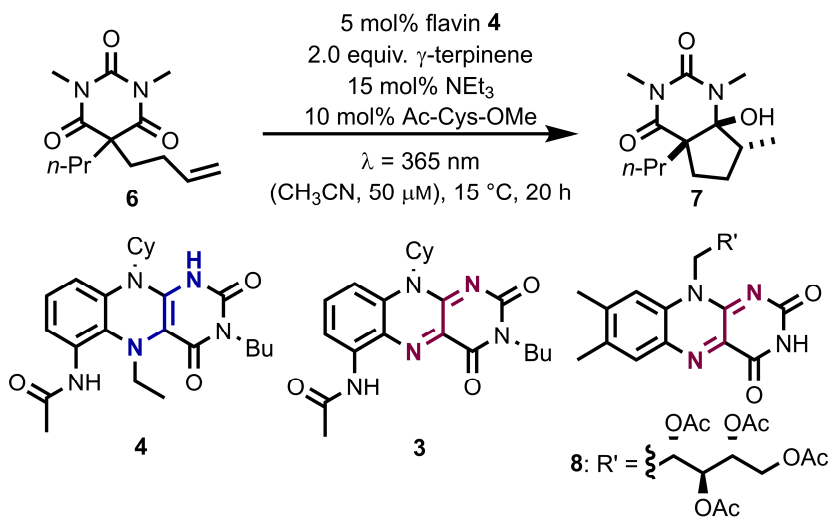

$\begin{array}{ccc}\text { Entry } & \text { Deviation from standard conditions } & \text { Yield }^{\mathrm{a}} \\ 1 & \text { None } & 90 \% \\ 2 & \text { No NEt }_{3} & \text { nd } \\ 3 & \text { No } \gamma \text {-terpinene } & <5 \% \\ 4 & \text { No Ac-Cys-OMe } & 39 \% \\ 5 & \text { No flavin catalyst } & \text { nd } \\ 6 & \text { No irradiation } & \text { nd } \\ 7 & \text { With } 4.0 \text { equiv. NEt } 3 & \text { quant. } \\ 8^{c} & 10 \text { mol\% (Ac-Cys-OMe) } 2 & \text { quant. } \\ 9^{c} & 5 \text { mol\% flavin catalyst } 3 & 46 \\ 10^{c} & 5 \text { mol\% flavin catalyst } 8 & 88\end{array}$

a: Measured by NMR spectroscopy with internal standard. b: Isolated yield on $0.1 \mathrm{mmol}$ substrate scale. c: With 4.0 equiv. $\mathrm{NEt}_{3}$. nd: Not detected.

Leaving out the amine base resulted in no conversion. This is in analogy to the enzymatic process, which relies on the deprotonated cofactor $\mathrm{FADH}^{-}($Entry 2$) .{ }^{2 \mathrm{f}}$ The $\gamma$-terpinene is required as a sacrificial reductant and only trace product is formed in its absence due to irreversible catalyst oxidation (Entry 3). Cysteine improves the yield significantly but is not essential for catalysis (Entry 4). We also verified that catalyst and irradiation are necessary (Entries 5,6). Increasing amounts of triethylamine led to quantitative conversion (Entry 7) and other bases such as quinuclidine are also suitable (see SI). Cystine and cysteine perform equally well, which implies that the former is efficiently reduced under the reaction conditions as well (Entry 8). Under our optimized conditions, in-situ reduced quinoid flavin 3 and even (-)-riboflavin tetraacetate $\mathbf{8}$ also led to product formation (Entries 9,10), which highlights the applicability of our method.

According to our mechanistic proposal, the catalytic reaction is initiated by a one-electron reduction of barbiturate 6, very similar to the first step in DNA photolyase (Figure 4). Our flavin catalysis proceeds relatively slowly with a quantum yield of $\Phi=1.2 \cdot 10^{-3}$ (see SI) and in line with this observation, no significant fluorescence quenching 
of $4^{-*}$ occurs upon addition of substrate 6 presumably due to inefficient electron transfer. The intermediate ketyl

110 radical next undergoes rapid 5-exo-trig cyclization to anion $7^{--}$which is further reduced and protonated to yield 111 bicyclic product 7. Hydrogen atom transfer from $\gamma$-terpinene (BDE of 1,4-cyclohexadiene is $\left.77 \mathrm{kcal} \mathrm{mol}^{-1}\right)^{19} \mathrm{result}^{1}$ 112 ing in para-cymene via 9 seems plausible for reduction of both intermediate $\mathbf{7}^{-\boldsymbol{}}$ and semiquinone $\mathbf{4}^{\circ}$. In agreement 113 with this line of events, hydroquinone 4 is regenerated quickly and is the only catalyst species we observe when 114 conducting the reaction in an NMR tube under inert conditions (see SI). During the catalytic reaction, photoexcita115 tion of intermediate $4^{\circ}$ will also occur given the typical absorption properties of flavin semiquinones. ${ }^{20}$ These species 116 are also strong reductants in the excited state, ${ }^{7}$ however, we have not observed any quinoid flavin under our reaction conditions by NMR. Catalytic amounts of cysteine as a hydrogen atom donor ensure rapid conversion of the carboncentered radicals.

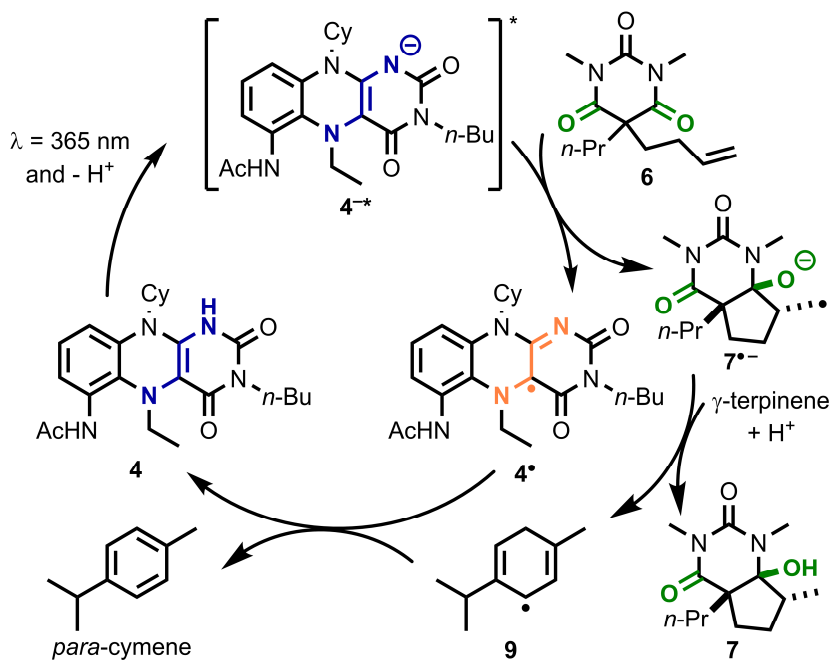

121 Figure 4. Simplified mechanistic proposal for the flavin-catalyzed reduction of barbituric acid derivative 6. The carbonyl groups, one of which is initially reduced, are highlighted in green.

124 Gratifyingly, flavin catalysis also allows efficient reductive conversion of a series of barbituric acid derivatives 125 (Figure 5). The 5-exo-trig cyclization is initiated with different substituted alkene side chains (10-12) and di- as 126 well as tri-substitution is tolerated. With allene substrates, terminal alkene product $\mathbf{1 3}$ is formed. An analogous 127 cyclization forming six-membered rings is also possible, but bicycle $\mathbf{1 4}$ was obtained as a mixture of diastereomers. 128 With terminal alkynes, 5-exo-dig cyclization results in exocyclic alkene $\mathbf{1 5 .}$ 

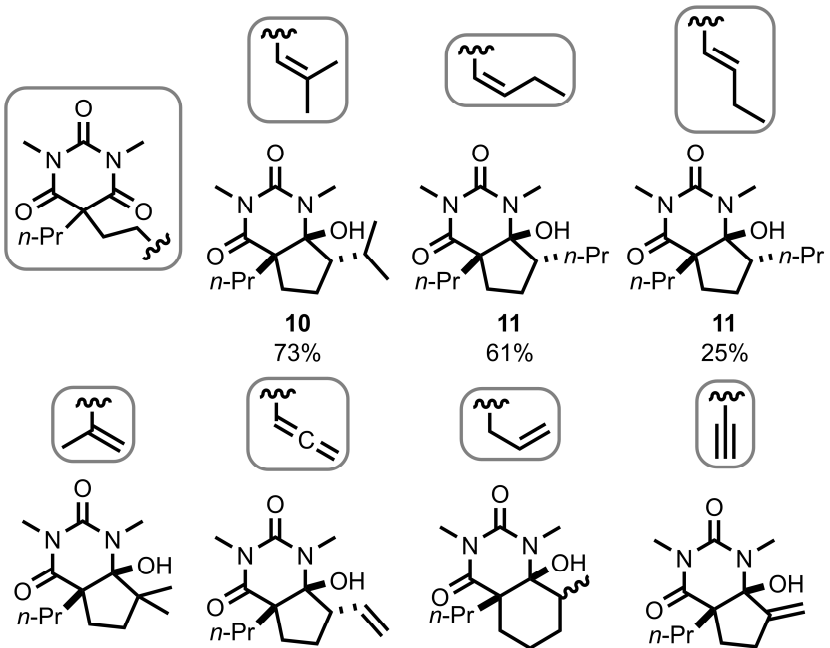

12
$40 \%$



$68 \%$

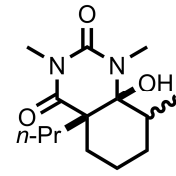

14

$77 \%$ (d.r. $2: 1$ )
11
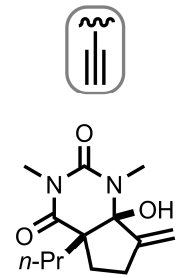

15

$77 \%$

131 Figure 5. Application of the flavin catalysis to a variety of barbituric acid derivatives. Reaction conditions: $5 \mathrm{~mol} \%$ $1324\left(10 \mathrm{~mol} \%\right.$ for product 11), 2.0 equiv. $\gamma$-terpinene, $\lambda=365 \mathrm{~nm},\left(\mathrm{CH}_{3} \mathrm{CN}\right), 15 \mathrm{~h}$. Yields were determined by NMR 133 spectroscopy with internal standard.

We next probed for potential differences between flavin-mediated reductions and conventional reactions using $\mathrm{SmI}_{2}$. In this context, barbituric acid derivative 16 was chosen, which was reported to result in pinacol coupling product 17 upon reduction with $\mathrm{SmI}_{2}$ (Figure 6) ${ }^{21}$ Here, three carbonyl sites compete for the one-electron reduction, and the formation of the pinacol product was rationalized based on a favored ( $E=-2.2 \mathrm{~V} v s$. SCE) initial reduction of a barbituric acid carbonyl group compared to the ketone $\left(E<-2.5 \mathrm{~V} v s\right.$. SCE) ${ }^{21}$ In contrast, when applying flavin catalyst 4, no pinacol product was obtained and secondary alcohol 18 was formed instead. This reactivity difference could be explained by substrate chelation when using $\mathrm{SmI}_{2}$, which allows flavin 4 to leave the carbonyl groups in the heterocycle fully intact.

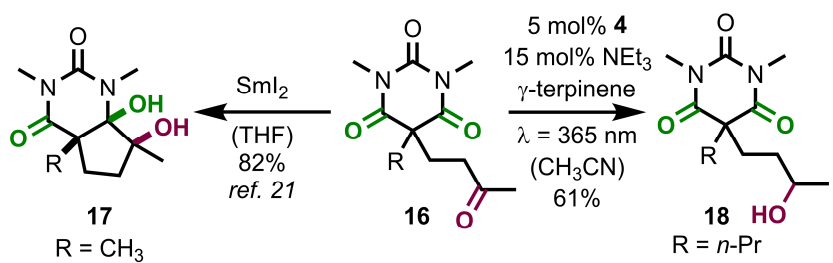

Figure 6. Reduction of ketone 16 shows distinct differences between $\mathrm{SmI}_{2}$-mediated and flavin-catalyzed reactions.

In summary, we report reduced, molecular flavins as catalysts in organic transformations. While so far such reduced flavins have been exclusively used as $\mathrm{O}_{2}$-reductants, our combination of the essential oil reductant $\gamma$-terpinene, photochemical excitation, and mild basic conditions allow the conversion of other, useful organic substrates. Inspired by DNA photolyase, we focused on barbituric acid derivatives in this study but envision broad applicability 
to other substrate classes based on the strong reducing power of excited flavins. Additionally, we observed differences between traditional metal reductants and flavin catalysts, which stimulate our continuing search for other challenging transformations as well as chiral catalyst versions for stereoselective reductions.

\section{Supporting Information}

The Supporting Information (PDF) contains experimental procedures, analytical data for all new compounds, additional experiments, and NMR data. The X-ray crystallographic coordinates for $\mathbf{4}$ are deposited at the Cambridge Crystallographic Data Center (CCDC) under deposition number CCDC 2126062.

\section{Acknowledgments}

161 The Fonds der Chemischen Industrie (FCI, Ph.D. Fellowship to A.W and Liebig Fellowship to G.S) is gratefully 162 acknowledged. R.F. thanks the Studienstiftung des Deutschen Volkes for a Ph.D. fellowship. The project was funded 163 by the Deutsche Forschungsgemeinschaft (DFG, German Research Foundation) - TRR 325 - 444632635. We thank 164 K. Rickmeyer and L. Niederegger (research group of Prof. C. Hess) for their assistance with cyclic voltammetry, 165 D. P. Schwinger and J. Großkopf for spectroscopic measurements, and O. Ackermann for HPLC analyses. G.S. is 166 supported by the Emmy Noether Programme (DFG, STO 1175/3-1) and the Technical University of Munich (Junior 167 Fellow Programme). He is very grateful to Prof. T. Bach for his continuous support.

Notes

170 The authors declare no competing financial interests.

\section{References}

(1) (a) Mansoorabadi, S. O.; Thibodeaux, C. J.; Liu, H.-w., The Diverse Roles of Flavin Coenzymes - Nature's Most Versatile Thespians. J. Org. Chem. 2007, 72, 6329-6342. (b) Joosten, V.; van Berkel, W. J. H., Flavoenzymes. Curr. Opin. Chem. Biol. 2007, 11, 195-202. (c) Walsh, C. T.; Wencewicz, T. A., Flavoenzymes: Versatile catalysts in biosynthetic pathways. Nat. Prod. Rep. 2013, 30, 175-200.

(2) (a) Sancar, A., Structure and function of DNA photolyase. Biochemistry 1994, 33, 2-9. (b) Kao, Y.-T.; Saxena, C.; Wang, L.; Sancar, A.; Zhong, D., Direct observation of thymine dimer repair in DNA by photolyase. Proc. Natl. Acad. Sci. USA 2005, 102, 16128-16132. (c) Kao, Y.-T.; Saxena, C.; He, T.-F.; Guo, L.; Wang, L.; Sancar, A.; Zhong, D., Ultrafast Dynamics of Flavins in Five Redox States. J. Am. Chem. Soc. 2008, 130, 13132-13139. (d) Harbach, P. H. P.; Schneider, M.; Faraji, S.; Dreuw, A., Intermolecular Coulombic Decay in Biology: The Initial Electron Detachment from FADH ${ }^{-}$in DNA Photolyases. J. Phys. Chem. Lett. 2013, 4, 943-949. (e) Tan, C.; Liu, Z.; Li, J.; Guo, X.; Wang, L.; Sancar, A.; Zhong, D., The molecular origin of high DNA-repair efficiency by photolyase. Nat. Commun. 2015, 6, 7302. (f) Zhong, D., Electron Transfer Mechanisms of DNA Repair by Photolyase. 
Annu. Rev. Phys. Chem. 2015, 66, 691-715. (g) Sancar, A., Mechanisms of DNA Repair by Photolyase and Excision

186 Nuclease (Nobel Lecture). Angew. Chem. Int. Ed. 2016, 55, 8502-8527. (h) Zhang, M.; Wang, L.; Shu, S.; Sancar, 187 A.; Zhong, D., Bifurcating electron-transfer pathways in DNA photolyases determine the repair quantum yield. 188 Science 2016, 354, 209-213.

(3) Scannell, M. P.; Fenick, D. J.; Yeh, S.-R.; Falvey, D. E., Model Studies of DNA Photorepair: Reduction Potentials of Thymine and Cytosine Cyclobutane Dimers Measured by Fluorescence Quenching. J. Am. Chem. Soc. 1997, 119, 1971-1977.

(4) (a) Rokita, S. E.; Walsh, C. T., Flavin and 5-deazaflavin photosensitized cleavage of thymine dimer: a model of in vivo light-requiring DNA repair. J. Am. Chem. Soc. 1984, 106, 4589-4595. (b) Pac, C.; Miyake, K.; Masaki, Y.; Yanagida, S.; Ohno, T.; Yoshimura, A., Flavin-photosensitized monomerization of dimethylthymine cyclobutane dimer: remarkable effects of perchloric acid and participation of excited-singlet, triplet, and chain-reaction pathways. J. Am. Chem. Soc. 1992, 114, 10756-10762. (c) Yasuda, M.; Nishinaka, Y.; Nakazono, T.; Hamasaki, T.; Nakamura, N.; Shiragami, T.; Pac, C.; Shima, K., Photochemistry of Flavins in Micelles: Specific Effects of Anionic Surfactants on the Monomerization of Thymine Cyclobutane Dimers Photosensitized by Tetra-O-acyl Riboflavins. Photochem. Photobiol. 1998, 67, 192-197.

(5) Hartman, T.; Cibulka, R., Photocatalytic Systems with Flavinium Salts: From Photolyase Models to Synthetic Tool for Cyclobutane Ring Opening. Org. Lett. 2016, 18, 3710-3713.

(6) (a) Clayman, P. D.; Hyster, T. K., Photoenzymatic Generation of Unstabilized Alkyl Radicals: An Asymmetric Reductive Cyclization. J. Am. Chem. Soc. 2020, 142, 15673-15677. (b) Huang, X.; Wang, B.; Wang, Y.; Jiang, G.; Feng, J.; Zhao, H., Photoenzymatic enantioselective intermolecular radical hydroalkylation. Nature 2020, 584, 6974. (c) Sandoval, B. A.; Clayman, P. D.; Oblinsky, D. G.; Oh, S.; Nakano, Y.; Bird, M.; Scholes, G. D.; Hyster, T. K., Photoenzymatic Reductions Enabled by Direct Excitation of Flavin-Dependent "Ene"-Reductases. J. Am. Chem. Soc. 2021, 143, 1735-1739. (d) Gao, X.; Turek-Herman, J. R.; Choi, Y. J.; Cohen, R. D.; Hyster, T. K., Photoenzymatic Synthesis of $\alpha$-Tertiary Amines by Engineered Flavin-Dependent “Ene”-Reductases. J. Am. Chem. Soc. 2021, 143, 19643-19647.

(7) Graml, A.; Neveselý, T.; Jan Kutta, R.; Cibulka, R.; König, B., Deazaflavin reductive photocatalysis involves excited semiquinone radicals. Nat. Commun. 2020, 11, 3174.

(8) (a) Imada, Y.; Naota, T., Flavins as organocatalysts for environmentally benign molecular transformations. Chem. Rec. 2007, 7, 354-361. (b) Cibulka, R., Artificial Flavin Systems for Chemoselective and Stereoselective Oxidations. Eur. J. Org. Chem. 2015, 915-932.

(9) For general reviews on the use of molecular flavins in catalysis see: (a) de Gonzalo, G.; Fraaije, M. W., Recent Developments in Flavin-based Catalysis. ChemCatChem 2013, 5, 403-415. (b) Rehpenn, A.; Walter, A.; Storch, G., Molecular Editing of Flavins for Catalysis. Synthesis 2021, 53, 2583-2593.

(10) Szostak, M.; Sautier, B.; Spain, M.; Behlendorf, M.; Procter, D. J., Selective Reduction of Barbituric Acids Using $\mathrm{SmI}_{2} / \mathrm{H}_{2} \mathrm{O}$ : Synthesis, Reactivity, and Structural Analysis of Tetrahedral Adducts. Angew. Chem. Int. Ed. 2013, 52, 12559-12563. 
(11) For related, reductive cyclization reactions of barbituric acid derivatives with $\mathrm{SmI}_{2}$ see: (a) Huang, H.-M.;

Procter, D. J., Radical-Radical Cyclization Cascades of Barbiturates Triggered by Electron-Transfer Reduction of

Amide-Type Carbonyls. J. Am. Chem. Soc. 2016, 138, 7770-7775. (b) Huang, H.-M.; Procter, D. J., Dearomatizing

Radical Cyclizations and Cyclization Cascades Triggered by Electron-Transfer Reduction of Amide-Type Carbonyls. J. Am. Chem. Soc. 2017, 139, 1661-1667.

(12) Kemal, C.; Chan, T. W.; Bruice, T. C., Reaction of ${ }^{3} \mathrm{O}_{2}$ with dihydroflavins. 1. N3,5-Dimethyl-1,5-dihydrolumiflavin and 1,5-dihydroisoalloxazines. J. Am. Chem. Soc. 1977, 99, 7272-7286.

(13) Eberlein, G.; Bruice, T. C., The chemistry of a 1,5-diblocked flavin. 2. Proton and electron transfer steps in the reaction of dihydroflavins with oxygen. J. Am. Chem. Soc. 1983, 105, 6685-6697.

(14) (a) Hasford, J. J.; Kemnitzer, W.; Rizzo, C. J., Conformational Effects on Flavin Redox Chemistry. J. Org. Chem. 1997, 62, 5244-5245. (b) Reibenspies, J. H.; Guo, F.; Rizzo, C. J., X-ray Crystal Structures of Conformationally Biased Flavin Models. Org. Lett. 2000, 2, 903-906.

(15) (a) Tauscher, L.; Ghisla, S.; Hemmerich, P., NMR.-Study of Nitrogen Inversion and Conformation of 1,5Dihydro-isoalloxazines ('Reduced Flavin'). Studies in the flavin series, XIX. Communication. Helv. Chim. Acta 1973, 56, 630-644. (b) Zheng, Y.-J.; Ornstein, R. L., A Theoretical Study of the Structures of Flavin in Different Oxidation and Protonation States. J. Am. Chem. Soc. 1996, 118, 9402-9408. (c) Rodríguez-Otero, J.; MartínezNúñez, E.; Peña-Gallego, A.; Vázquez, S. A., The Role of Aromaticity in the Planarity of Lumiflavin. J. Org. Chem. 2002, 67, 6347-6352.

(16) Walsh, J. D.; Miller, A.-F., Flavin reduction potential tuning by substitution and bending. J. Mol. Struct. Theochem 2003, 623, 185-195.

(17) Selected examples for using $\gamma$-terpinene as a sacrificial reductant in photochemical transformations: (a) Schweitzer-Chaput, B.; Horwitz, M. A.; de Pedro Beato, E.; Melchiorre, P., Photochemical generation of radicals from alkyl electrophiles using a nucleophilic organic catalyst. Nat. Chem. 2019, 11, 129-135. (b) de Pedro Beato, E.; Mazzarella, D.; Balletti, M.; Melchiorre, P., Photochemical generation of acyl and carbamoyl radicals using a nucleophilic organic catalyst: applications and mechanism thereof. Chem. Sci. 2020, 11, 6312-6324.

(18) For the BDE of cysteine, see: Roux, M. V.; Foces-Foces, C.; Notario, R.; Ribeiro da Silva, M. A. V.; Ribeiro da Silva, M. d. D. M. C.; Santos, A. F. L. O. M.; Juaristi, E., Experimental and Computational Thermochemical Study of Sulfur-Containing Amino Acids: 1-Cysteine, 1-Cystine, and 1-Cysteine-Derived Radicals. S-S, S-H, and C-S Bond Dissociation Enthalpies. J. Phys. Chem. B 2010, 114, 10530-10540.

(19) Gao, Y.; DeYonker, N. J.; Garrett, E. C.; Wilson, A. K.; Cundari, T. R.; Marshall, P., Enthalpy of Formation of the Cyclohexadienyl Radical and the C-H Bond Enthalpy of 1,4-Cyclohexadiene: An Experimental and Computational Re-Evaluation. J. Phys. Chem. A 2009, 113, 6955-6963.

(20) Kabir, M. P.; Orozco-Gonzalez, Y.; Gozem, S., Electronic spectra of flavin in different redox and protonation states: a computational perspective on the effect of the electrostatic environment. Phys. Chem. Chem. Phys. 2019, 255 $21,16526-16537$. 
256 (21) Kise, N.; Tuji, T.; Sakurai, T., Stereoselective intramolecular coupling of barbituric acids with aliphatic ke257 tones and $O$-methyl oximes by electroreduction: radical cyclization mechanism supported by DFT study. Tetrahe258 dron Lett. 2016, 57, 1790-1793. 